\title{
TRUE STRESS AND POISSON'S RATIO OF TENDONS DURING LOADING
}

\author{
Claudio Vergari ${ }^{\mathrm{a},}$, Philippe Pourcelot ${ }^{\mathrm{a}}$, Laurène Holden ${ }^{\mathrm{a}}$, Bérangère Ravary-Plumioën ${ }^{\mathrm{a}}$, \\ Guillaume Gerard ${ }^{\mathrm{a}}$, Pascal Laugier ${ }^{\mathrm{b}}$, David Mitton ${ }^{\mathrm{c}}$ and Nathalie Crevier-Denoix ${ }^{\mathrm{a}}$
}

\begin{abstract}
Excessive axial tension is very likely involved in the aetiology of tendon lesions, and the most appropriate indicator of tendon stress state is the true stress, the ratio of instantaneous load to instantaneous cross-sectional area (CSA). Difficulties to measure tendon CSA during tension often led to approximate true stress by assuming that CSA is constant during loading (i.e. by the engineering stress) or that tendon is incompressible, implying a Poisson's ratio of 0.5 , although these hypotheses have never been tested.

The objective of this study was to measure tendon CSA variation during quasi-static tensile loading, in order to assess the true stress to which the tendon is subjected and its Poisson's ratio. Eight equine superficial digital flexor tendons (SDFT, about 30 $\mathrm{cm}$ long) were tested in tension until failure while the CSA of each tendon was measured in its metacarpal part by means of a linear laser scanner. Axial elongation and load were synchronously recorded during the test.

CSA was found to linearly decrease with strain, with a mean decrease at failure of $-10.7 \pm 2.8 \%$ (mean \pm standard deviation). True stress at failure was 7.1 to $13.6 \%$ higher than engineering stress, while stress estimation under the hypothesis of incompressibility differed from true stress of -6.6 to $2.3 \%$. Average Poisson's ratio was $0.55 \pm 0.12$ and did not significantly vary with load.

From these results on equine SDFT it was demonstrated that tendon in axial quasistatic tension can be considered, at first approximation, as an incompressible material.
\end{abstract}

Keywords: Tendon; Cross-sectional area; Poisson's ratio; True stress; Mechanical testing. 


\section{Introduction}

Mechanical characterization of tendons has been a subject of research for a long time (e.g., Abrahams, 1967; Rigby et al., 1959; Vanbrocklin and Ellis, 1965), but the lack of devices capable of reliably measuring cross-sectional area (CSA) of specimens during mechanical testing has limited many studies. For instance, the true stress (i.e. the ratio of instantaneous load to instantaneous CSA) to which the tendon is subjected during loading has often been approximated by making assumptions on tendon CSA variation. Nevertheless, it is considered the proper indicator of the stress state of soft tissues undergoing large deformations (Vincent, 1990), a situation which is incriminated in the aetiology of tendon injuries (Patterson-Kane and Firth, 2009; Webbon, 1977).

The most common approximation of true stress is engineering stress (defined as the ratio of instantaneous load to initial CSA), which assumes a constant CSA during tension. An alternative is the estimation of the instantaneous CSA under the hypothesis of constant volume during loading (i.e. incompressibility). This approximation has been applied, for instance, to human and equine tendons (Abrahams, 1967), collagen matrices (Roeder et al., 2002) and fibrils (Eppell et al., 2006).

Incompressibility implies a Poisson's ratio (PR, the negative ratio of transverse to axial true strain) of 0.5 , a value commonly accepted for various soft biological tissues because of their high water content (water being nearly incompressible). However, only a few PR measurements have been performed, in rat (Cheng and Screen, 2007) and sheep (Lynch et al., 2003), and they are in excess of 0.5. Recently, Reese et al. (2010) suggested that such high values (0.8 and $2.98 \pm 2.59$, respectively) may depend on the tendon microstructure, which is characterized by a helical organization of fibrils within crimped fibers.

Many non-contact techniques to measure CSA have been described in the literature, such as laser based systems (Lee and Woo, 1988; Moon et al., 2006; Revel et al., 2003). Although accurate and not invasive, these systems require long acquisition times which are incompatible with dynamic or quasi-static measurements. A faster laser system (still requiring $20 \mathrm{~s}$ for each acquisition) was developed and applied to measure bovine tendons undergoing tensile loads (Pokhai et al., 2009); however, this system requires precise centering of the specimen in the measured zone, a task not easily performed with long specimens that can undergo tensile loads up to $20 \mathrm{kN}$.

A linear scanner laser system, adapted to measurements of biological specimens CSA at rest or during mechanical testing, capable of performing fast acquisitions and not needing precise specimen centering, was recently developed (Vergari et al., 2010a). It was applied to preliminary CSA measurements of equine tendons (Vergari et al., 2010b), which are frequently injured and represent an excellent model for human tendon research.

The objectives of the present study were: 1 . to apply the above mentioned technique to measure the instantaneous CSA during quasistatic loading until failure on eight equine superficial digital flexor tendons (SDTFs), 2. to use these CSA values to calculate true stress and evaluate the error introduced by the hypotheses underlying its most common approximations (negligible CSA decrease or incompressibility), and 3. to determine the tendons' PR and its evolution during loading, in order to add to the understanding of tendon's mechanical properties.

\section{Materials and methods}

\subsection{Tendons}

Eight SDFTs were isolated from the left forelimb of horses of various breeds, killed at the abattoir for reasons unrelated to this study (Table 1). The limbs, cut at the carpo-metacarpal junction, were stored frozen at $-20^{\circ} \mathrm{C}$ in plastic bags until the day of the experiment. As no information on the horses' bodyweight was available, measurement of the minimal cannon bone (Os metacarpale III) 


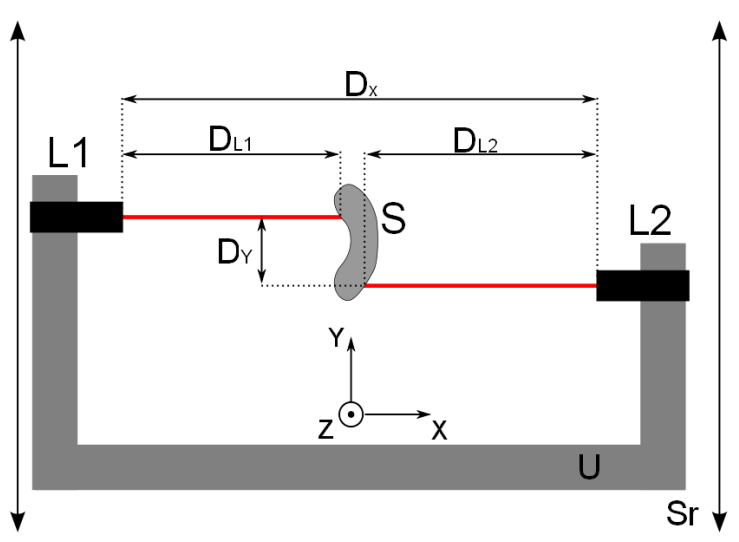

Figure 1. Schematic top view of the linear laser scanner. Lasers (L1 and L2) are mounted on a U shaped plate for synchronization and slide along two horizontal linear guides (not in the presented scheme) along the $y$ direction for the whole sweeping range (Sr) The specimen $(\mathrm{S})$ is placed in the middle with its main axis parallel to the $\mathrm{z}$ axis. Characteristic distances are reported: Dx and Dy are, respectively, the distance between lasers and the offset imposed to avoid mutual interference; DL1 and DL2, the distances measured by each laser between the specimen surface and themselves.

transverse diameter was performed for each horse (Table 1), as this parameter is reported to be correlated to the horse's weight (Rhoad, 1929).

\subsection{Cross-sectional area measurements}

CSA was assessed, statically and during loading, with a linear laser scanner described elsewhere (Vergari et al., 2010a). Briefly, it consists of two CCD laser reflectance devices (Keyence LK-G82, Courbevoie, France) mounted facing each other on two horizontal linear guides where they can be manually and synchronously slid to sweep, with their laser beams, a measuring zone of about $10 \mathrm{~cm}$.

The specimen is vertically placed in the measuring zone, as shown in Figure 1, so that the two lasers can measure the distance between the specimen surface and themselves when sweeping the entire measuring range.

The lasers horizontal position along the $y$ axis (Figure 1) is measured with a string potentiometer and an offset is imposed along the $y$ direction between the two lasers to avoid mutual interference. With reference to the distances definition of Figure 1, the coordinates of the points forming the tendon perimeter can be obtained with the expressions:

$$
\begin{aligned}
& \left\{\begin{array}{l}
x_{L 1}=-\left(D_{x} / 2-D_{L 1}\right) \\
x_{L 2}=\left(D_{x} / 2-D_{L 2}\right)
\end{array}\right. \\
& \left\{\begin{array}{l}
y_{L 1}=y_{p} \\
y_{L 2}=y_{L 1}-D_{y}
\end{array}\right.
\end{aligned}
$$

where the coordinates subscripts L1 and L2 denote the points measured by laser 1 and laser 2 , respectively, while $\mathrm{y}_{\mathrm{p}}$ is the position measured by the string potentiometer. CSA can then be calculated integrating the linear interpolations between perimeter points.

Since the measured section moves vertically during loading, the system measuring block is mounted on a cart sliding on a vertical column so it can be moved to follow the measured area (Vergari et al., 2010a).

Table 1. Characteristics of the 8 superficial digital flexor tendons tested (x: missing data, F: female, M: male).

\begin{tabular}{|cccccc}
\hline \hline Tendon & Horse age [years] & $\begin{array}{c}\text { Horse } \\
\text { gender }\end{array}$ & $\begin{array}{c}\text { Cannon bone } \\
\text { diameter [mm] }\end{array}$ & Breed & $\begin{array}{c}\text { Duration of } \\
\text { preservation } \\
\text { (freezing) } \\
\text { [months] }\end{array}$ \\
\hline 1 & 12 & F & 44.9 & Thoroughbred & 8.5 \\
2 & 15 & F & 38.0 & Standardbred & 9.4 \\
3 & 3 & M & 39.6 & Thoroughbred & 9.2 \\
4 & $\mathrm{x}$ & $\mathrm{x}$ & 39.5 & x & 11.6 \\
5 & 9 & $\mathrm{M}$ & 40.6 & Standardbred & 11.9 \\
6 & 14 & $\mathrm{~F}$ & 44.4 & Standardbred & 12.0 \\
7 & 13 & $\mathrm{~F}$ & 41.7 & French Warmblood & 8.8 \\
8 & 4 & $\mathrm{M}$ & 46.3 & &
\end{tabular}




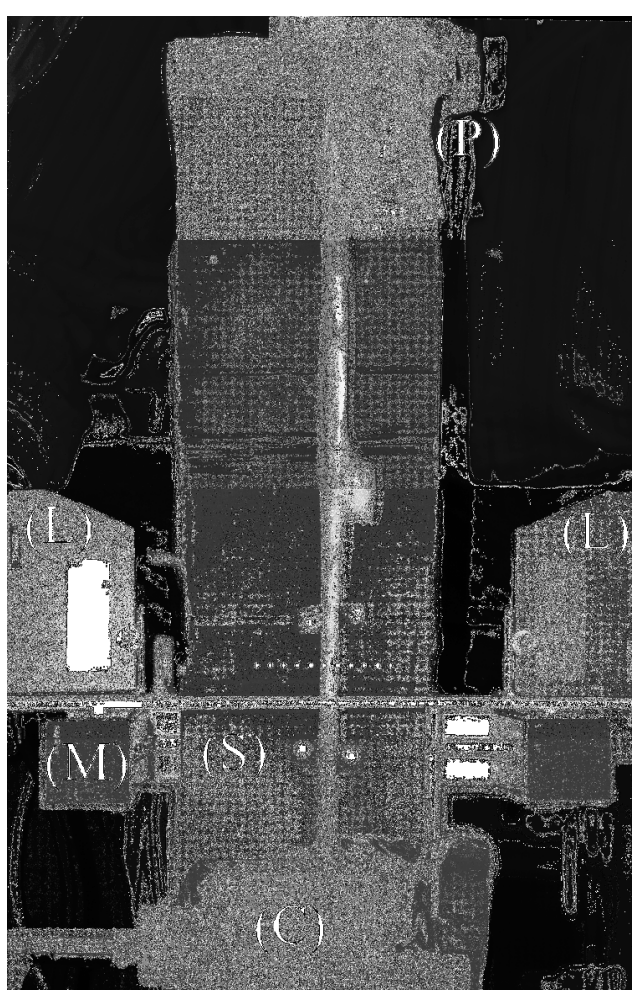

Figure 2. A superficial digital flexor tendon mounted on the testing machine along with the linear scanner laser. The phalanx $(\mathrm{P})$ is maintained by metal rods and the proximal end of the tendon is blocked by the cryo-jaw (C), thermally protected by means of a plastic bag. Scotchlite markers (S) are glued to small foam cubes held in place by needles pinned across the tendon. The measuring block $(\mathrm{M})$, holding the CCD lasers $(\mathrm{L})$ can be moved vertically to follow the tendon cross-section of interest (dotted line, corresponding to the measured cross-sectional area) during loading.

\subsection{Experimental protocol}

Each tendon (about $30 \mathrm{~cm}$ in length) was mounted on a testing machine $(10 / \mathrm{MH}$, MTS Systems Corporation, Eden Prairie, MN USA) by clamping its proximal end in a cryo-jaw (Riemersma and Schamhardt, 1982), while its distal insertion on the middle phalanx was left intact. The phalanx was maintained by means of metal rods that allowed free sagittal rotation and lateral sliding, inducing self-alignment of the tendon with load and, therefore, the verticality of the specimen (Figure 2).

The region of interest (ROI) was defined in the distal metacarpal part of the tendon (Crevier et al., 1996) and its proximal limit was about $5 \mathrm{~cm}$ from the frozen portion of the tendon to avoid any effect on the tendon mechanical properties. Two needles were inserted across the tendon in the dorso-palmar direction, perpendicular to the tendon main axis and distant of about $10 \mathrm{~cm}$ from each other; the extremities of each needle were then equipped with circular scotchlite markers (Figure 2). A digital camera (Nikon D100) was placed at the same height of the ROI, with its axis orthogonal to the plane formed by the four markers and at $2 \mathrm{~m}$ from the ROI.

A static tensile load of $50 \mathrm{~N}$ was applied, to avoid sagging, then the tendon initial crosssectional area of interest (CSAOI) was defined in the ROI and measured with the linear laser scanner. Ten successive measures were performed and averaged to determine the initial CSA. During the last of these measurements, a photo was taken with the camera to assess the initial length of the ROI $\left(\mathrm{L}_{0}\right)$, by measuring the distance between the middle points of both pairs of markers in the picture.

The tendon was loaded at a constant speed of $20 \mathrm{~mm} / \mathrm{min}$, while CSA measures were performed continuously until failure of the tendon. For each CSA acquisition a picture of the ROI was taken. Length $(\mathrm{L})$ of the ROI during loading was measured with a custom-made software tracking the markers on each image. Axial engineering strain was then calculated as $\varepsilon_{A}=\left(L-L_{0}\right) / L_{0}$ for each acquisition.

Load information, available through the analogical output of the testing machine, was digitized together with the lasers and string potentiometer outputs with a National Instruments card (USB-6210, National Instruments, Austin, TX USA).

\subsection{Poisson's ratio computation}

PR for materials undergoing large deformations, i.e. when strain exceeds $1 \%$ (Rees, 2006), is defined as the negative ratio of transverse to axial true strain (Smith et al., 1999):

$v=-\frac{\ln \left(1+\varepsilon_{T}\right)}{\ln \left(1+\varepsilon_{A}\right)}$ 




Figure 3. Cross-sectional area variation of tendon \#3 undergoing tensile load. Linear approximation $\left(\mathrm{R}^{2}=0.92\right)$ has a slope of $-0.87 \mathrm{~mm}^{2} / \%$ of strain (corresponding to $-0.71 \%$ of the initial area $/ \%$ of strain).

where $\varepsilon_{\mathrm{T}}$ and $\varepsilon_{\mathrm{A}}$ are the transverse and axial engineering strains, respectively.

An infinitesimal square element in the CSAOI, orthogonal to the tendon main axis and of initial side $\delta \mathrm{x}_{0}$, undergoes a plane deformation when tendon is subjected to loading. Eq. (3) can be rearranged as:

$\ln \left(\delta x / \delta x_{0}\right)=\ln \left[\left(1+\varepsilon_{A}\right)^{-v}\right]$

where $\delta \mathrm{x}$ is the side of the square element in the deformed configuration. It can be calculated from Eq. (4):

$$
\delta x=\delta x_{0}\left(1+\varepsilon_{A}\right)^{-v}
$$

This is valid under the hypothesis of isotropy or, more appropriately for tendons, transverse isotropy. In the latter case the PR relative to the appropriate strains couple (transversal to axial, instead of axial to transversal, or transversal to transversal, the three ratios coinciding in isotropic materials) has to be used.

The CSA of the square in the deformed configuration, $\delta \mathrm{A}$, is:

$$
\begin{aligned}
& \delta A=\delta x^{2}=\delta x_{0}{ }^{2}\left(1+\varepsilon_{A}\right)^{-2 v}= \\
& =\delta A_{0}\left(1+\varepsilon_{A}\right)^{-2 v}
\end{aligned}
$$

where $\delta A_{0}$ is the initial CSA of the square. By simple summation of all the elements composing the tendon CSA, the instantaneous CSA can be expressed as:

$$
A=A_{0}\left(1+\varepsilon_{A}\right)^{-2 v}
$$

where $A$ and $A_{0}$ are, respectively, the instantaneous and initial tendon CSA. The linear laser scanner used in the present study was specifically designed to perform CSA measurements, so this information was used to calculate tendons PR with the following rearrangement of Eq. (7):

$v=-0.5 \frac{\ln \left(A / A_{0}\right)}{\ln \left(1+\varepsilon_{A}\right)}$

\subsection{True stress approximation}

True stress is defined as the ratio of instantaneous load to instantaneous CSA but, knowing the initial CSA, its value can be approximated by assuming that tendon is incompressible, implying a PR of 0.5. The instantaneous CSA for an incompressible material, $\mathrm{A}_{\mathrm{I}}$, can be expressed as:

$$
A_{I}=\left\lfloor A_{0}\left(1+\varepsilon_{A}\right)^{-2 v}\right\rfloor_{V=0.5}=A_{0} /\left(1+\varepsilon_{A}\right)
$$

Thus, instantaneous CSA can be evaluated knowing its initial value and the axial engineering strain.

The error introduced by this true stress approximation can be estimated, if the actual $\mathrm{PR}$ is known, from the ratio of Eq. (7) to Eq. (9):

$$
\begin{aligned}
& 100 \cdot\left(\frac{\text { True Stress Approximation }}{\text { True Stress }}-1\right) \%= \\
& =100 \cdot\left[\frac{F / A_{I}}{F / A}-1\right] \%=100 \cdot\left[\left(1+\varepsilon_{A}\right)^{1-2 v}-1\right] \%
\end{aligned}
$$

where $\mathrm{F}$ is the load applied to the tendon.

\section{Results}

\subsection{Cross-sectional area and axial strain}

Figure 3 shows a representative example of CSA decrease with axial engineering strain. All CSA data could be well described with linear 


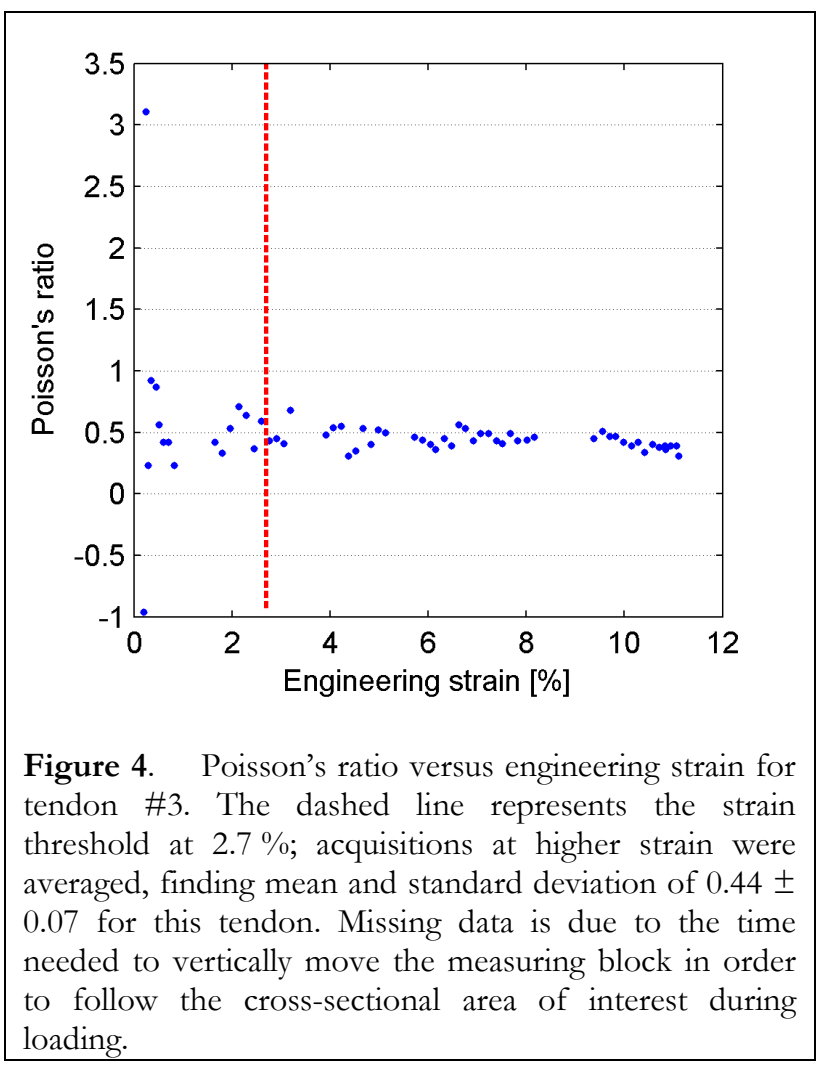

approximations $\left(\mathrm{R}^{2}>0.72\right)$, obtaining percentage variation in the range between -0.71 and $-1.25 \%$ of initial area $\%$ of axial strain (mean $-0.96 \pm 0.17$ ). The maximal CSA variation found, among the tendons that failed, is $-13.6 \%$ for tendon \#5. The actual axial strain rate of the tendon ROI was $4.9 \pm 1 \% / \mathrm{min}$ (mean \pm standard deviation, calculated on all specimens), with engineering strain of $11.8 \pm$ $1.5 \%$ at tendon failure.

\subsection{Poisson's ratio}

Since axial true strain data exhibited a rather simple shape when plotted versus stress, it has been approximated with polynomials for the subsequent calculations in order to minimize noise on PR values. However, raw CSA values were used to calculate PR, instead of a linear approximation for instance, to avoid introduction of artifactual shapes to the PR versus strain curves.

Figure 4 shows an example set of PR measurements. All sets appeared more affected by noise at low strain than at higher strain; moreover, measurements at high strain were not significantly correlated to load $(p>0.05)$. Since values of PRs are not much reliable at low strain, the measurements corresponding to an axial strain lower than $2.7 \%$ were discarded; this threshold was defined because it is the lowest limit to make 7 out of 8 datasets pass a normality test for small samples number (Lilliefors test with $\alpha=0.05)$. Tendon \#5 did not pass the normality test probably because it had quite noisy CSA measurements, due to irregularities present on its surface.

PR resulted to be between 0.42 and 0.77 , with a mean value of 0.55 (values determined with the $2.7 \%$ strain threshold); table 2 reports PR mean values and standard deviations for each tendon. No correlation was found between PR and available data on specimen characteristics, such as horse age, cannon bone minimal transverse diameter or freezing time, or structural characteristics such as initial CSA or breaking strength (tables 1 and 2).

\subsection{Stress}

Engineering stress at tendon failure was $7.1 \%$ to $13.6 \%$ smaller than the corresponding true stress (failure strength values are reported in Table 2); this range is a result of the minimal and maximal (respectively) CSA variation measured at tendon failure.

The error introduced by the true stress approximation over true stress can be estimated with Eq. (10): for an axial strain of $12 \%$ it yields an error of $-6.6 \%$ when the actual PR is 0.8 , and of $2.3 \%$ when it is 0.4 ; the error is almost linear with PR value. A comparative graph of true stress and true stress approximations is shown in Figure 5.

\section{Discussion}

By means of a non-contact method to measure CSA of soft tissues during mechanical testing, CSA, true stress and PR were assessed in equine tendons during loading.

Pokhai et al. (2009) reported a CSA decrease of $13.5 \pm 1.9 \%$ at $2.5 \%$ axial strain for bovine deep and superficial digital flexor tendons. In the present study, a CSA decrease of about $10.7 \pm 2.8 \%$ was measured at tendon failure 




Figure 5. Curves of true stress (TS), engineering stress (ES) and true stress approximated by evaluating cross-sectional area variation during loading under the hypothesis of incompressibility (ATS), implying a Poisson's ratio of 0.5 . Data is plotted against engineering strain and is relative to tendon \#3, which underwent tensile loading until failure. Final values are 132.6 MPa, 124.3 $\mathrm{MPa}$ and 139.3 MPa respectively, for TS, ES and ATS. The difference with TS is $-6.3 \%$ (ES versus. TS) and $+5 \%$ (ATS versus TS). Missing data is due to the time needed for the vertical displacement of the measuring block.

(i.e. at axial strain of $11.8 \pm 1.5 \%$, corresponding to a slope of $-0.96 \% / \%$ of axial strain). This large difference is likely due to the different protocols applied: Pokhai et al. performed continuous CSA acquisitions during loading, with acquisition time exceeding $20 \mathrm{~s}$, of longitudinal slices of tendon stained with white ink; besides, no definition of initial length and axial strain was given.

The present study is the first attempt to evaluate tendon PR directly from CSA measurements, while other studies were based on the measure of linear transverse deformation under the hypothesis of small deformations. Mean PR was evaluated to be 0.8 , with some data exceeding 1 , for fascicles teased from rat tails proximal ends (Cheng and Screen, 2007). These results were obtained by measuring the deformation of a grid photobleached on the samples, which underwent tensile loads increments. In another study (Lynch et al., 2003) ovine flexor tendons were cut in rectangular samples and tested at different strain-rates in axial and transverse direction, finding mean PRs of $2.98 \pm 2.59$ and $0.488 \pm 0.653$, respectively. No information on the precision of the techniques was given by these authors.
In the present study, PR of 8 equine SDFTs was found in the range between 0.42 and 0.77 (mean 0.55 ). These values were obtained without the hypothesis of small deformations, appropriately for strains exceeding $1 \%$. However, it was estimated that the introduction of the small deformation hypothesis $\left(v \approx-\varepsilon_{T} / \varepsilon_{A}\right)$ would lead to an underestimation of the actual PR of only $-4.9 \%$.

The precision of PR measurement in the present study was estimated at $15.1 \%$ (with $95 \%$ confidence interval) when it is considered constant and is averaged on 44 measures during axial deformation (which is the mean number of acquisitions after the $2.7 \%$ strain threshold). This result was obtained by evaluating the error propagation with the differential method described in Deming (1950); the multivariate variance of PR was approximated from Eq. (8) in the worst case scenario (i.e. by setting the working point at the $2.7 \%$ strain threshold; the standard deviation of the CSA dynamic measurements was evaluated assuming that CSA decreases linearly with load). The source of noise below the threshold can be found in Eq. (8): at low strain both the numerator and denominator are nearly zero, so their ratio becomes very sensitive to noise.

While isotropic media cannot have PRs higher than 0.5 , a limit imposed by thermodynamic considerations, orthotropic or transversely isotropic materials (such as tendons) are commonly found with values higher than 1 (Lempriere, 1968). A model describing the microstructural tendon behavior in axial loading has been proposed by Reese et al. (2010). This model is capable of predicting PRs across the isotropic limit, depending on mechanical and geometrical parameters (the ratio of the fiber modulus to the matrix modulus, the angle of the fiber crimps and the pitch of fibril helical arrangement). In particular, these authors obtained PRs which are almost constant with axial strain for fibers with crimp angles between 10 and $20^{\circ}$. Moreover, small PRs $(<1)$ have been found for non-helical fibers. Consistently with the relatively small and constant PR we measured, a helical arrangement of fibrils has never been described, to our knowledge, for 


\begin{tabular}{|c|c|c|c|c|c|c|c|}
\hline \multirow{2}{*}{ Tendon } & \multirow{2}{*}{$\begin{array}{c}\text { Initial area } \\
{[\mathrm{mm} 2]} \\
(\text { mean } \pm \mathrm{SD})\end{array}$} & \multicolumn{2}{|c|}{$\begin{array}{c}\text { Final deformation } \\
{[\%]}\end{array}$} & \multirow{2}{*}{$\begin{array}{l}\text { Poisson's ratio } \\
\text { (mean } \pm \mathrm{SD})\end{array}$} & \multicolumn{3}{|c|}{ Failure stress $[\mathrm{MPa}]$} \\
\hline & & $\begin{array}{c}\text { CSA } \\
\text { decrease }\end{array}$ & $\begin{array}{l}\text { Engineering } \\
\text { strain }\end{array}$ & & Engineering & True & Approximated \\
\hline 1 & $111.70 \pm 0.56$ & -10.3 & 9.1 & $0.51 \pm 0.08$ & $\mathrm{x}$ & $\mathrm{x}$ & $\mathrm{x}$ \\
\hline 2 & $113.77 \pm 1.81$ & -12.5 & 11.4 & $0.62 \pm 0.15$ & 140.89 & 160.94 & 156.62 \\
\hline 3 & $122.58 \pm 0.99$ & -6.3 & 11.1 & $0.44 \pm 0.07$ & 124.25 & 132.65 & 138.05 \\
\hline 4 & $118.18 \pm 1.04$ & -9.7 & 12.4 & $0.62 \pm 0.14$ & 105.00 & 116.26 & 118.06 \\
\hline 5 & $110.41 \pm 1.40$ & -13.6 & 14.0 & $0.48 \pm 0.18$ & 128.66 & 148.91 & 146.67 \\
\hline 6 & $117.24 \pm 0.79$ & -7.1 & 8.2 & $0.42 \pm 0.05$ & $\mathrm{x}$ & $\mathrm{x}$ & $\mathrm{x}$ \\
\hline 7 & $99.83 \pm 0.73$ & -10.6 & 8.3 & $0.77 \pm 0.10$ & $\mathrm{x}$ & $\mathrm{x}$ & $\mathrm{x}$ \\
\hline 8 & $107.52 \pm 0.70$ & -11.5 & 10.0 & $0.51 \pm 0.08$ & 122.52 & 138.43 & 134.73 \\
\hline $\begin{array}{l}\text { Mean } \\
\pm \mathrm{SD}\end{array}$ & $112.7 \pm 7.0$ & $-10.2 \pm 2.5$ & $10.6 \pm 2.0$ & $0.55 \pm 0.12$ & $124.3 \pm 12.9$ & $139.4 \pm 16.9$ & $138.8 \pm 14.4$ \\
\hline
\end{tabular}

equine SDFT, while crimp angles have been found between 10.5 and $14.6^{\circ}$ (Patterson-Kane et al., 1997).

A PR across 0.5 has interesting implications on tendon behavior: a value smaller than 0.5 implies an increase in volume during loading, corresponding, if weight losses are neglected, to a decrease in density. On the contrary, a value higher than 0.5 implies a volume reduction and an increase in density. Still, the mean PR for all tendons tested in the present study was 0.55 , so tendon in axial loading can be considered, with good approximation, as an incompressible material. The small volume variations measured are probably due to internal rearrangements of the tendon structures and, as suggested by Lynch et al. (2003), water loss, although no macroscopic water leak was observed. In fact, our results might underestimate the actual tendon PR since the limbs were stored frozen before the experiments, which might have caused some dehydration, although all precautions were adopted to avoid it.

The difference between engineering and true stress corresponds to the CSA percentage variation, leading to a non-negligible maximal underestimation of $-13.6 \%$ at tendon failure. True stress approximation with the hypothesis that tendon is an incompressible material can yield underestimated or overestimated values, depending on the actual tendon PR. The error was estimated between $-6.6 \%$ and $2.3 \%$. Thus, approximating true stress by assuming that tendon is incompressible gives better results than calculating engineering stress.

This study reported equine tendon CSA decrease and true stress during quasi-static loading until rupture. It was demonstrated that approximating true stress with the assumption of tendon incompressibility induces smaller errors than adopting the engineering stress. PR, a parameter which adds to the mechanical characterization of soft tissues and is useful for modeling and simulation, was assessed for equine tendons and found near the limit of incompressibility.

\section{Conflict of interest statement}

The authors have no conflicts of interest to disclose.

\section{Acknowledgments}

We are grateful to the Direction Générale de l'Enseignement et de la Recherche (French Ministry of Agriculture), the Région BasseNormandie and the INRA (Institut National de la Recherche Agronomique) for financial support. 


\section{References}

Abrahams, M., 1967. Mechanical behaviour of tendon in vitro. A preliminary report. Medical and Biological Engineering and Computing 5, 433-443.

Cheng, V. W. T., Screen, H. R. C., 2007. The micro-structural strain response of tendon. Journal of Materials Science 42, 8957-8965.

Crevier, N., Pourcelot, P., Denoix, J. M., Geiger, D., Bortolussi, C., Ribot, X., Sanaa, M., 1996. Segmental variations of in vitro mechanical properties in equine superficial digital flexor tendons. American Journal of Veterinary Research 57, 1111-1117.

Deming, W. E. 1950. Some Theory of Sampling, John Wiley and Sons, Inc., New York, pp. 127-131.

Eppell, S. J., Smith, B. N., Kahn, H., Ballarini, R., 2006. Nano measurements with micro-devices: mechanical properties of hydrated collagen fibrils. Journal of the Royal Society Interface 3, 117-121.

Lee, T. Q., Woo, S. L., 1988. A new method for determining cross-sectional shape and area of soft tissues. Journal of Biomechanical Engineering 110, 110-114.

Lempriere, B. M., 1968. Poisson's ratio in orthotropic materials. American Institute of Aeronautics and Astronautics Journal 6, 2226-2227.

Lynch, H. A., Johannessen, W., Wu, J. P., Jawa, A., Elliott, D. M., 2003. Effect of fiber orientation and strain rate on the nonlinear uniaxial tensile material properties of tendon. Journal of Biomechanical Engineering - Transactions of the ASME 125, 726-731.

Moon, D. K., Abramowitch, S. D., Woo, S. L., 2006. The development and validation of a chargecoupled device laser reflectance system to measure the complex cross-sectional shape and area of soft tissues. Journal of Biomechanics 39, 3071-3075.

Patterson-Kane, J. C., Firth, E. C., 2009. The pathobiology of exercise-induced superficial digital flexor tendon injury in Thoroughbred racehorses. The Veterinary Journal 181, 79-89.

Patterson-Kane, J. C., Firth, E. C., Goodship, A. E., Parry, D. A., 1997. Age-related differences in collagen crimp patterns in the superficial digital flexor tendon core region of untrained horses. Australian Veterinary Journal 75, 39-44.

Pokhai, G. G., Oliver, M. L., Gordon, K. D., 2009. A new laser reflectance system capable of measuring changing cross-sectional area of soft tissues during tensile testing. Journal of Biomechanical Engineering 131, 094504.

Rees, D. W. A. 2006. Basic Engineering Plasticity: An Introduction with Engineering and Manufacturing Applications, Butterworth-Heinemann, Oxford, pp. 40-41.

Reese, S. P., Maas, S. A., Weiss, J. A., 2010. Micromechanical models of helical superstructures in ligament and tendon fibers predict large Poisson's ratios. Journal of Biomechanics 43, 1394 1400.

Revel, G. M., Scalise, A., Scalise, L., 2003. Measurement of stress-strain and vibrational properties of tendons. Measurement Science \& Technology 14, 1427-1436.

Rhoad, A. O., 1929. Relation between conformation and pulling ability of draft horses. Journal of Animal Science 1929, 182-188.

Riemersma, D. J., Schamhardt, H. C., 1982. The cryo-jaw, a clamp designed for in vitro rheology studies of horse digital flexor tendons. Journal of Biomechanics 15, 619-620.

Rigby, B. J., Hirai, N., Spikes, J. D., Eyring, H., 1959. The mechanical properties of rat tail tendon. The Journal of General Physiology 43, 265-283.

Roeder, B. A., Kokini, K., Sturgis, J. E., Robinson, J. P., Voytik-Harbin, S. L., 2002. Tensile mechanical properties of three-dimensional type I collagen extracellular matrices with varied microstructure. Journal of Biomechanical Engineering 124, 214-222.

a USC INRA-ENVA, Biomécanique et Pathologie Locomotrice du Cheval, Ecole Nationale

Vétérinaire d'Alfort, 7 avenue du Général de Gaulle, 94704 Maisons-Alfort cedex, France

b UPMC Univ Paris 6, UMR CNRS7623, LIP, Paris, F-75005 France

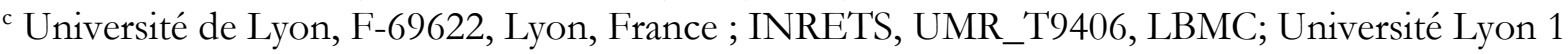

${ }^{*}$ Corresponding author. E-mail address: c.vergari@gmail.com (C. Vergari) 
Smith, C., Wootton, R., Evans, K., 1999. Interpretation of experimental data for Poisson's ratio of highly nonlinear materials. Experimental Mechanics 39, 356-362.

Vanbrocklin, J. D., Ellis, D. G., 1965. A study of the mechanical behavior of toe extensor tendons under applied stress. Archives of Physical Medicine and Rehabilitation 46, 369-373.

Vergari, C., Pourcelot, P., Holden, L., Ravary-Plumioën, B., Laugier, P., Mitton, D., Crevier-Denoix, N., 2010a. A Linear Laser Scanner to Measure Cross-Sectional Shape and Area of Biological Specimens During Mechanical Testing. Journal of Biomechanical Engineering 132, 105001 105008.

Vergari, C., Pourcelot, P., Holden, L., Ravary-Plumioën, B., Laugier, P., Mitton, D., Crevier-Denoix, N., 2010b. Measurement of cross-sectional area variations of five equine superficial digital flexor tendons during tension. Computer Methods in Biomechanics and Biomedical Engineering 13, 143-144.

Vincent, J. F. V. 1990. Basic theory of elasticity and viscoelasticity. In: Fisher, D. (Ed.) Structural Biomaterials. Princeton, Princeton University Press. pp. 5-7.

Webbon, P. M., 1977. A post mortem study of equine digital flexor tendons. Equine Veterinary Journal 9, 61-67. 